\title{
Effects of integrin $\alpha 5 \beta 1$ on the proliferation and migration of human aortic vascular smooth muscle cells
}

\author{
YAN SONG, XIAOYU QIN, HANJIE WANG, RENYING MIAO, \\ YONGGAN ZHANG, CHAOFENG MIAO and ZIFAN WANG \\ Department of Vascular Surgery, The First Affiliated Hospital of Zhengzhou University, \\ Zhengzhou, Henan 450052, P.R. China
}

Received June 9, 2014; Accepted April 30, 2015

DOI: $10.3892 / \mathrm{mmr} .2015 .4649$

\begin{abstract}
Integrin (ITG) $\alpha 5 \beta 1$ is a dominant fibronectin receptor that is abundantly expressed on the surface of vascular smooth muscle cells (VSMCs). However, the association between integrin $\alpha 5 \beta 1$ and the proliferation and migration of VSMCs has yet to be elucidated. The aim of the present study was to characterize the roles of ITG $\alpha 5$ and ITG $\beta 1$ in the proliferation and migration of VSMCs, and to determine the effects of ITG $\alpha 5 \beta 1$ on integrin-linked kinase (ILK) and focal adhesion kinase (FAK) mRNA expression. Lentiviral expression vectors as well as RNA interference vectors of ITG $\alpha 5$ and ITG $\beta 1$ were successfully constructed and transfected into VSMCs to obtain ITG $\alpha 5$ - and ITG $\beta 1$-overexpressing or -silenced cells, respectively. Cell cycle distribution, proliferation and migration were analyzed in the transfected VSMCs in order to clarify the roles of ITG $\beta 1$ and ITG $\alpha 5$ in the proliferation and migration of VSMCs. ITG $\beta 1$ was markedly associated with the proliferation and migration of VSMCs, and FAK was shown to be involved in the signaling pathways of ITG $\beta 1$. ITG $\alpha 5$ did not exert any effects on VSMCs. The results of the present study may provide a possible therapeutic target for the prevention and treatment of early vascular disease associated with VSMCs.
\end{abstract}

\section{Introduction}

Proliferation and migration of vascular smooth muscle cells (VSMCs) from the tunica media to the tunica intima is an early event in response to vascular injury, including atherosclerosis and angioplasty-induced restenosis (1). Interactions between the cells and surrounding extracellular matrix (ECM) have important roles in numerous cellular processes, including differentiation, proliferation, migration, cytoskeletal organization and

Correspondence to: Dr Yan Song, Department of Vascular Surgery, The First Affiliated Hospital of Zhengzhou University, 40 Daxue Road, Zhengzhou, Henan 450052, P.R. China

E-mail: songyan101112@sina.com

Key words: $\alpha 5 \beta 1$ integrin, proliferation, migration, lentivirus, RNA interference, vascular smooth muscle cells survival (2). Fibronectin is an extracellular glycoprotein that has important roles in cell adhesion, migration, growth and differentiation (3). Integrin (ITG) $\alpha 5 \beta 1$ is a dominant fibronectin receptor comprised of the $\alpha 5$ and $\beta 1$ subunits, which is known to be abundantly expressed on the surface of VSMCs (4). ITG $\beta$ is able to activate signaling pathways, including the focal adhesion kinase (FAK), Src and Ras/extracellular signal-regulated kinase (ERK) pathways, which are dependent on the interactions between various extracellular matrix (ECM) proteins and ITG (5). FAK and its autonomously expressed C-terminal inhibitor FAK-related non-kinase (FRNK), are important regulators of VSMC spreading and migration (6). Integrin-linked kinase (ILK) is a downstream mediator of ITG $\beta 1$ activity. ILK activity has previously been shown to be associated with numerous cell functions (7). It has previously been suggested that ILK has a role in the cell adhesion, proliferation, migration, matrix remodeling and survival of various types of tissue and cells (7).

The role of ITG $\alpha 5 \beta 1$ in the proliferation and migration of VSMCs has not yet been reported. The present study aimed to explore the regulatory effects of ITG $\alpha 5 \beta 1$ on the proliferation and migration of VSMCs, investigate alterations to the FAK and ILK signaling pathways and understand the underlying mechanisms. The present study constructed a lentiviral expression vector of ITG $\alpha 5 \beta 1$ as well as a small interfering RNA (siRNA) lentiviral vector of ITG $\alpha 5 \beta 1$ in order to obtain VSMC with ITG $\alpha 5 \beta 1$ overexpression and knockdown, respectively. The proliferation, migration, cell cycle distribution and mRNA expression levels of transfected VSMCs were then analyzed.

\section{Materials and methods}

Reagents. Fetal bovine serum (FBS) and RPMI 1640 were purchased from Gibco (Thermo Fisher Scientific, Waltham, MA, USA). Trypsin was obtained from HyClone (GE Healthcare, Logan, UT, USA). RNase and propidium iodide (PI) were obtained from Sigma-Aldrich (St. Louis, MO, USA). pGEM-T vector was purchased from Tiangen Biotech Co., Ltd. (Beijing, China). The lentiviral expression vector pLenti, and lentivirus packaging plasmids pLP1 and pLP/VSVG were a generous gift from by Dr Huijun Zhi (Department of Microbiology and Immunology, Uniformed Services University of the Health Sciences, National Institutes of Health, Bethesda, MA, USA). pCMV-SPORT6-ITG $\alpha 5$ and pCMV-SPORT6-ITG $\beta 1$ were 
Table I. siRNAs used in the present study.

\begin{tabular}{ll} 
siRNA & Sequence \\
\hline IA11 & 5'-GATCCGGACCAGGAAGCTATTTCTTTCAAGAGAAGAAATAGCTTCCTGGTCCTTTTTTC-3' \\
IA12 & 5'-TCGAGAAAAAAGACCAGGAAGCTATTTCTTCTCTTGAAAGAAATAGCTTCCTGGTCCG-3' \\
IA21 & 5'-GATCCGCTATGTCACCATCCTTAATTCAAGAGATTAAGGATGGTGACATAGCTTTTTTC-3' \\
IA22 & 5'-TCGAGAAAAAAGCTATGTCACCATCCTTAATCTCTTGAATTAAGGATGGTGACATAGCG-3' \\
IB11 & 5'-GATCCGAGCCACAGACATTTACATTTCAAGAGAATGTAAATGTCTGTGGCTCTTTTTTC-3' \\
IB12 & 5'-TCGAGAAAAAAGAGCCACAGACATTTACATTCTCTTGAAATGTAAATGTCTGTGGCTCG-3' \\
IB21 & 5'-GATCCGTCAGCAGTAGGAACATTATTCAAGAGATAATGTTCCTACTGCTGACTTTTTC-3' \\
IB22 & 5'-TCGAGAAAAAAGTCAGCAGTAGGAACATTATCTCTTGAATAATGTTCCTACTGCTGACG-3'
\end{tabular}

siRNA, small interfering RNA.

purchased from OriGene Technologies, Inc. (Rockville, MD, USA). The plasmid pRNAT-U6.2/Lenti was obtained from GenScript (Piscataway, NJ, USA). Restriction enzymes BamHI, XhoI, KpnI and MluI were purchased from Promega Corp. (Madison, WI, USA). Lipofectamine ${ }^{\circledR} 2000$ was purchased from Invitrogen (Thermo Fisher Scientific). ITG $\alpha 5$ mouse monoclonal antibody (cat. no. SC-166681), ITG $\beta 1$ monoclonal antibody (cat. no. SC-71386), GAPDH monoclonal antibody (cat. no. SC-137179) and horseradish peroxidase-conjugated goat anti-mouse immunoglobulin G (cat no. SC-2005) were purchased from Santa Cruz Biotechnology, Inc. (Dallas, TX, USA). All antibodies were diluted at a 1:300 ratio and all incubations were performed for $1 \mathrm{~h}$ at room temperature. ITG $\alpha 5$ and ITG $\beta 1$ reverse-transcription polymerase chain reaction (RT-PCR) kits and primers were purchased from Qiagen China (Beijing, China). The other reagents used in the present study were of analytical grade.

Cell culture. The 293FT cells were obtained from the Shanghai Institute of Cell Biology, Chinese Academy of Sciences (Shanghai, China). The cells were maintained in RPMI 1640 supplemented with $10 \%$ FBS. Human aortic VSMCs (no. PCS-100-021) were purchased from the American Type Culture Collection (Manassas, VA, USA) and were cultured in RPMI 1640 supplemented with 10\% FBS and antibiotics (100 IU/ml penicillin and $100 \mu \mathrm{g} / \mathrm{ml}$ streptomycin; all from Sigma-Aldrich) at $37^{\circ} \mathrm{C}$ in a $5 \% \mathrm{CO}_{2}$ atmosphere.

Construction of the lentiviral expression vector and siRNA lentiviral vector of ITG $\alpha 5$ and ITG $\beta 1$. The entire cDNA sequences of ITG $\alpha 5$ and ITG $\beta 1$ were amplified by PCR of pCMV-SPORT6-ITG $\alpha 5$ and pCMV-SPORT6-ITG $\beta 1$. The upstream and downstream primers both contained KpnI and $M l u I$ endonuclease sites. The cDNA was then ligated with the pGEM-T vector. The ligation products were transfected into Escherichia coli DH5a cells (Beijing Hua Yueyang Biotechnology Co. Ltd., Beijing, China), which were maintained at $37^{\circ} \mathrm{C}$. The positive recombinant clones pGEM-T-ITG $\alpha 5$ and pGEM-T-ITG $\beta 1$ were then selected and maintained in LB medium for $4 \mathrm{~h}$ at $37^{\circ} \mathrm{C}$. Subsequently, $1 \mu \mathrm{l}$ bacterial medium was used as a template and PCR was performed using the EconoTaq PLUS 2X Master Mix (Lucigen, Madison, WI, USA) with T7 (5'-TAATACGACTCACTATAGGGAGA-3') and SP6 (5'-CATACGATTTAGGTGACACTATAG-3') primers according to manufacturer's instructions. The positive clones were subjected to DNA sequencing by Shanghai Sengong Biotech (Shanghai, China). The cloning vector and the lentivirus were cut using $K p n \mathrm{I}$ and $M l u \mathrm{I}$ restriction endonucleases, following which they were ligated and transfected. Enzyme analysis and gene sequencing analysis were used to verify the accuracy of the recombinant vectors pLenti-ITG $\alpha 5$ and pLenti-ITG $\beta 1$.

According to the nucleotide sequence of the ITG $\alpha 5$ and ITG $\beta 1$ genes in GenBank (http://www.ncbi.nlm.nih. gov/genbank/) and the principles of siRNA design, two segment sequences were selected from each: 735-753 nt and 970-988 nt, and 600-618 nt and 1,283-1,301 nt, respectively. The effective siRNA sequences targeting ITG $\alpha 5$ and ITG $\beta 1$ were then designed using the siRNA Designer web tool from Promega Corp. and synthesized by Sengong Biotech. The full list of siRNAs and their sequences used in the present study is shown in Table I. Both ends of the hairpin target sequences contained BamHI and XhoI endonuclease sites. The cDNA containing both the sense and antisense strands of the targeting sequence was designed, synthesized and cloned into the pRNAT-U6.2/Lenti vector, which contained the $\mathrm{H} 1$ promoter and green fluorescent protein (GFP). The resulting lentiviral vectors containing ITG $\alpha 5$ or ITG $\beta 1$ siRNA were named pRNAT-U6.2/Lenti-si ITG $\alpha 5-1$, pRNAT-U6.2/Lenti-siITG $\alpha 5-2$, pRNAT-U6.2/Lenti-siITG $\beta 1-1$ and pRNAT-U6.2/Lenti-siITG $\beta 1$-2. Restriction endonuclease digestion and DNA sequencing were conducted to confirm the generation of the recombinant vectors. PCR and gene sequencing analysis were used to verify the accuracy of the recombinant vectors.

Lentivirus packaging plasmid mixtures containing Lentivirus-ITG $\alpha 5$ or Lentivirus-ITG $\beta 1$, or pRNAT-U6.2/Lenti-siITG $\alpha 5-1$,pRNAT-U6.2/Lenti-siITG $\alpha 5-2$, pRNAT-U6.2/Lenti-siITG $\beta 1$-1 orpRNAT-U6.2/Lenti-siITG $\beta 1-2$ were co-transfected into the $293 \mathrm{FT}$ cells. All transfections were performed using Lipofectamine 2000 (Invitrogen) according to the manufacturer's instructions. The titer of the viral stock solutions was assessed via quantification of the expression levels of GFP as previously described (8).

VSMC transfection. Recombinant lentiviruses were transfected into the VSMCs in order to establish the following 
Table II. Sequence details of individual pairs of primers.

\begin{tabular}{lll}
\hline Gene & \multicolumn{1}{c}{ Sense } & \multicolumn{1}{c}{ Antisense } \\
\hline ILK1 & 5'-CTGGCAGCCAGTCATGGACAC-3' & 5'-ATGCTGACAAGGGCCCCATTT-3' \\
FAK & 5'-TTGCGGAGAATATGGCTGACCTAA-3' & 5'-TGGTATTGATGGCAAAGCCCGTTC-3' \\
ITG $\alpha 5$ & 5'-AGTGCACCCCCATTGAATTTG-3' & 5'-GAACTGTTGCCCCGAACCACT-3' \\
ITG $\beta 1$ & 5'-ACAGCAGTTGGTTTTGCGATT-3' & 5'-TCCAATTCTGAAGTCCGAAGT-3' \\
GAPDH & 5'-GGGAAGGTGAAGGTCGGAGTC-3' & 5'-CCCACTTGATTTTGGAGGGAT-3' \\
\hline
\end{tabular}

A

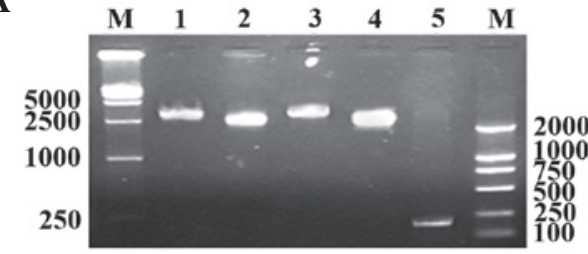

C

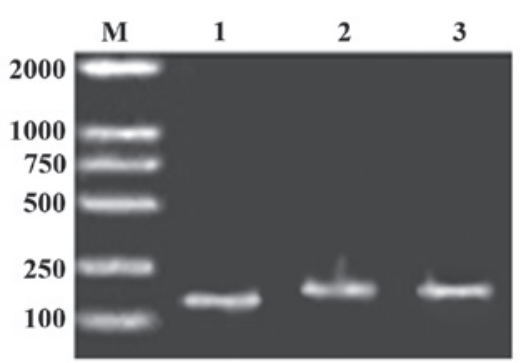

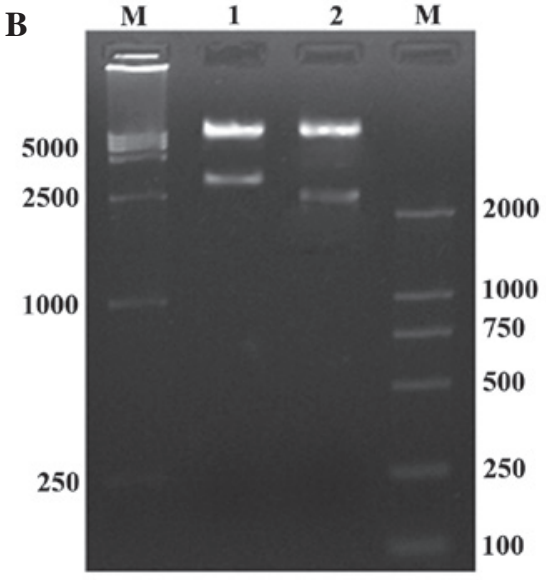

Figure 1. Target genes ITG $\alpha 5$ and ITG $\beta 1$, and recombinant clones. (A) PCR cloning of ITG $\alpha 5$ and ITG $\beta 1$ genes. M, marker; lane 1, ITG $\alpha 5$; lane 2, ITG $\beta 1$; lane 3, pGEM-ITG $\alpha$ 5; lane 4, pGEM-ITG $\beta 1$; lane 5, control vector pGEM-T. (B) Double digestion of recombinant clones. M, marker; lane 1, pLent-ITG $\alpha$ 5; lane 2, pLent-ITG $\beta 1$. (C) siRNA gene recombinant clones. M, marker; lane 1, pRNAT-U6.2/Lenti; lane 2, pRNAT-U6.2/Lenti-siITG $\alpha 5$; lane 3, pRNAT-U6.2/Lenti-siITG $\beta 1$. ITG, integrin; siRNA, small interfering RNA.

cell lines with upregulated and downregulated ITG $\alpha 5$ and ITG $\beta 1$ gene expression: Lentivirus-ITG $\alpha 5$, Lentivirus-ITG $\beta 1$, pRNAT-U6.2/Lenti-siITG $\alpha 5-1$,pRNAT-U6.2/Lenti-siITG $\alpha 5-2$, pRNAT-U6.2/Lenti-siITG $\beta 1$ 1, pRNAT-U6.2/Lenti-siITG $\beta 1-2$, pLentiGFP empty vector and pRNAT-U6.2/Lenti empty. Lipofectamine 2000 (Invitrogen) was used for all transfections according to the manufacturer's instructions. Screening with G418 (Sigma-Aldrich) was used to obtain stably transfected VSMCs. The transfected VMSC cell lines were named: ITGa5-overexpressing cell line (EX-ITGa5), ITG $\beta 1$-overexpressing cell line (EX-ITG $\beta 1)$, ITG $\alpha 5$-knockdown cell line (si-ITG $\alpha 5$ ), ITG $\beta 1-k$ nockdown cell line (si-ITG $\beta 1$ ), pLentiGFP empty vector-transfected cell line (Con-Ex) and pRNAT-U6.2/Lenti empty vector-transfected cell line (Con-si), respectively. Post-transfection, lentivirus-ITG $\alpha 5$ was transfected into the EX-ITG $\beta 1$ cell line in order to generate a cell line overexpressing both ITG $\alpha 5$ and ITG $\beta 1$ - this cell line was named D-EX. In addition, pRNAT-U6.2/Lenti-siITG $\alpha 5-1$ was transfected into the si-ITG $\beta 1-2$ cells in order to generate a cell line exhibiting both ITG $\alpha 5$ and ITG $\beta 1$ knockdown - this cell line was named D-si. Quantitative (q-PCR) and western blotting were used to detect the changes to ITG $\alpha 5$ and ITG $\beta 1$ gene and protein expression levels in all of the stably transfected cell lines $(4,9)$. Cell growth was observed using microscopy (Inverted microscope IX83; Olympus, Japan).
Proliferation assay. All experiments were conducted using the nine cell lines: EX-ITG $\alpha 5$, EX-ITG $\beta 1$, D-EX, si-ITG $\alpha 5$, si-ITG $\beta 1$, D-si, Con-Ex, Con-si and normal VSMCs. The nine cell lines were divided into three groups: Overexpressing groups, including EX-ITG $\alpha 5$, EX-ITG $\beta 1$ and D-EX; knockdown groups, including si-ITG $\alpha 5$, si-ITG $\beta 1$ and D-si; and control groups, including Con-Ex, Con-si and normal VSMCs.

All of the cell lines were cultured in 96-well microtiter plates (Corning, Inc., Corning, NY, USA) at $1 \times 10^{4}$ cells/well and incubated for five days at $37^{\circ} \mathrm{C}$. Subsequently, $20 \mu \mathrm{l}$ MTT (5 mg/ml; Jiemei Gene Pharmaceutical, Shanghai, China) was added to each well and incubated for $4 \mathrm{~h}$ at $37^{\circ} \mathrm{C}$. Following removal of the supernatant, $150 \mu$ l dimethyl sulfoxide (Jiemei Gene Pharmaceutical) was added to each well, and the absorbance values were measured using a microplate reader (iMark ${ }^{\mathrm{TM}}$ Microplate Absorbance reader; Bio-Rad Laboratories, Inc., Hercules, CA, USA) at $490 \mathrm{~nm}$ (9).

Migration assay. The migration assay was performed using a Transwell system, which allows cells to migrate through an $8-\mu \mathrm{m}$ pore polycarbonate membrane in a millicell $(10,11)$. Briefly, the cells were serum-starved for $24 \mathrm{~h}$ and then plated ( $1 \times 10^{4}$ cells/well) in serum-free medium (Gibco) in the upper chamber of a 24-well Transwell plate (Corning). The lower 


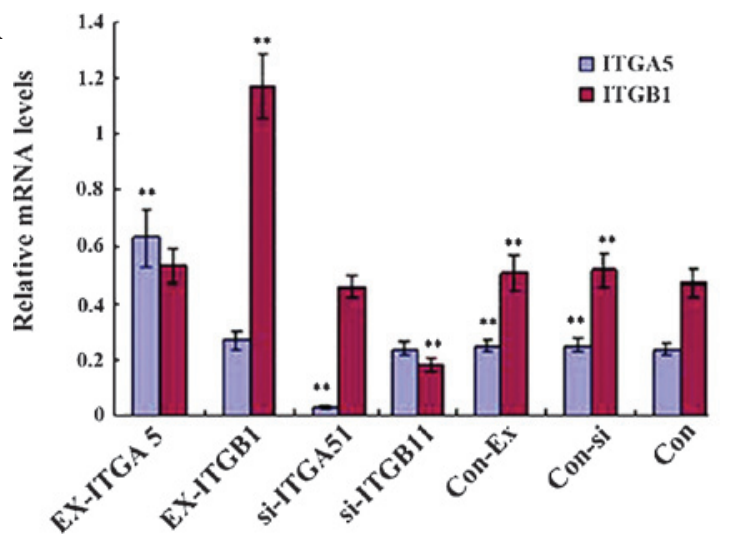

\section{B}

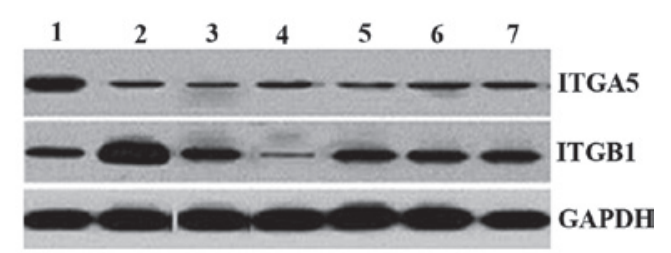

Figure 2. mRNA and protein expression levels of ITG $\alpha 5$ and ITG $\beta 1$ in the seven types of vascular smooth muscle cells. (A) mRNA expression levels of ITG $\alpha 5$ and ITG $\beta 1$ in the seven cell groups. (B) Protein expression levels of ITG $\alpha 5$ and ITG $\beta 1$ in the seven cell groups. Values are expressed as the mean \pm standard error of the mean $(n=3){ }^{* *} \mathrm{P}<0.05$ vs. Con. Lane 1, EX-ITG $\alpha 5$; lane 2, EX-ITG 11 ; lane 3, si-ITG $\alpha 5$; lane 4, si-ITG $\beta 1$; lane 5, Con-Ex; lane 6, Con-si; lane 7, Con. ITG, integrin; si, small interfering; Con, control; EX, overexpressing.
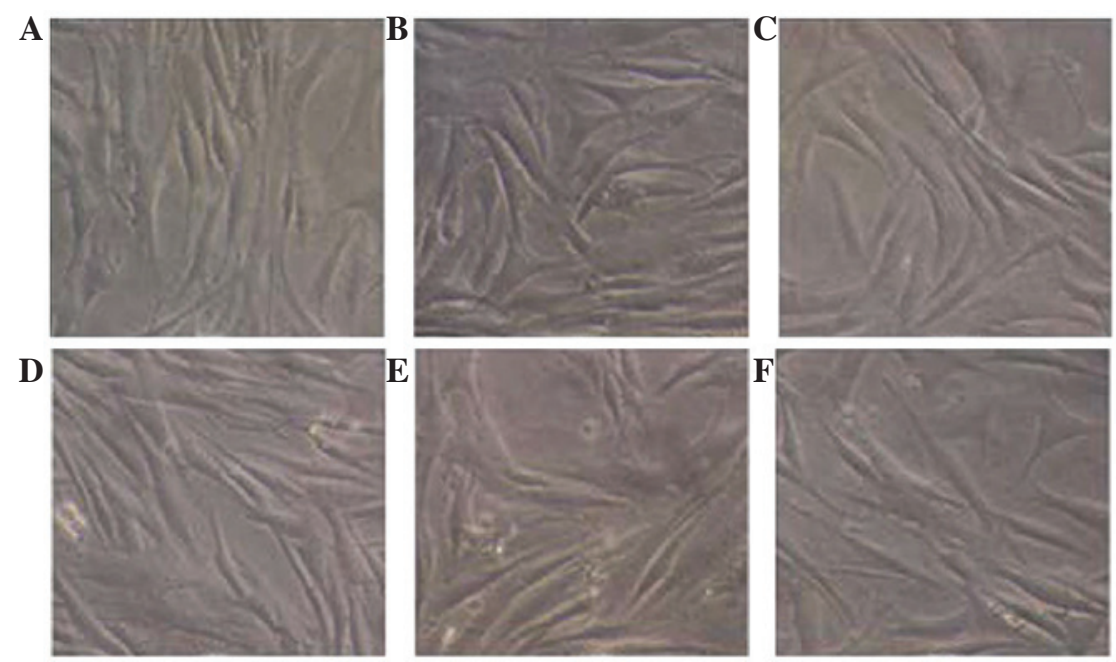

Figure 3. VSMCs were transfected with various lentiviruses (magnification, x400). (A) EX-ITG $\alpha 5$, VSMCs transfected with pLent-ITG $\alpha$. (B) EX-ITG $\beta 1$, VSMCs transfected with pLent-ITG $\beta 1$. (C) si-ITG $\alpha 5$, VSMCs transfected with pRNAT-U6.2/Lenti-siITG $\alpha 5$. (D) si-ITG $\beta 1$, VSMCs transfected with pRNAT-U6.2/Lenti-siITG 1 1. (E) Con-Ex, VSMCs transfected with pLentiGFP. (F) Con-si, VSMCs transfected with pRNAT-U6.2/Lenti. VSMC, vascular smooth muscle cell; ITG, integrin; si, small interfering; Con, control; GFP, green fluorescent protein; EX, overexpressing.

chamber was filled with $1.5 \mathrm{ml}$ medium supplemented with $500 \mu \mathrm{l}$ chemotactic factor (Jiemei Gene Pharmaceutical). After $48 \mathrm{~h}$, the cells that remained on the upper surface of the membrane were removed using a cotton swab, and the cells on the lower surface of the membrane were fixed with cold methanol for $15 \mathrm{~min}$ and stained with $0.2 \%$ crystal violet (Jiemei Gene Pharmaceutical). The cells that had migrated to the bottom of the membrane were visualized and counted using an inverted microscope. For each repetition, cells in four randomly selected fields were counted and averaged. Results were expressed as a ratio of the untreated group.

Cell cycle analysis. The cells were cultured in six-well culture plates for $24 \mathrm{~h}$ and incubated for a further $24 \mathrm{~h}$ in serum-free medium. The cells were then incubated in medium containing serum for another $48 \mathrm{~h}$. The cell cycle was assessed according to the intensity of PI staining, as described previously (12). Briefly, ethanol-fixed cells were centrifuged and washed twice with phosphate-buffered saline. Subsequently, cells were treated with $100 \mu 1$ RNase (Promega) for $30 \mathrm{~min}$ at $37^{\circ} \mathrm{C}$, followed by the addition of $100 \mu \mathrm{l}$ propidium iodide dye $(1 \mu \mathrm{g} / \mathrm{m} ; 1$ Sigma-Aldrich) and incubation for $30 \mathrm{~min}$ in the dark. Cell cycle analysis was performed using a flow Cytometer (FACS Aria II; BD Biosciences, Franklin Lakes, NJ, USA). Data were analyzed using Modfit LT software, version 3.2 (BD Biosciences).

$R T-q P C R$. Total RNA was isolated using a Total RNA Extraction kit (Qiagen, Hilden, Germany). Total RNA was then reverse-transcribed in a $20-\mu 1$ reaction solution containing Revert AID $^{\text {TM }}$ First Strand cDNA Synthesis kit (Fermentas; Thermo Fisher Scientific). The subsequent RT-qPCR, used to determine the expression levels of ILK and FAK, was conducted as described previously $(13,14)$. The sequences of the individual pairs of ILK, FAK, ITG $\alpha 5$, ITG $\beta 1$ and GAPDH primers (Qiagen) are presented in Table II. The PCR reactions were performed using the ABI StepOne Plus PCR system (Applied Biosystems; Thermo Fisher Scientific) 

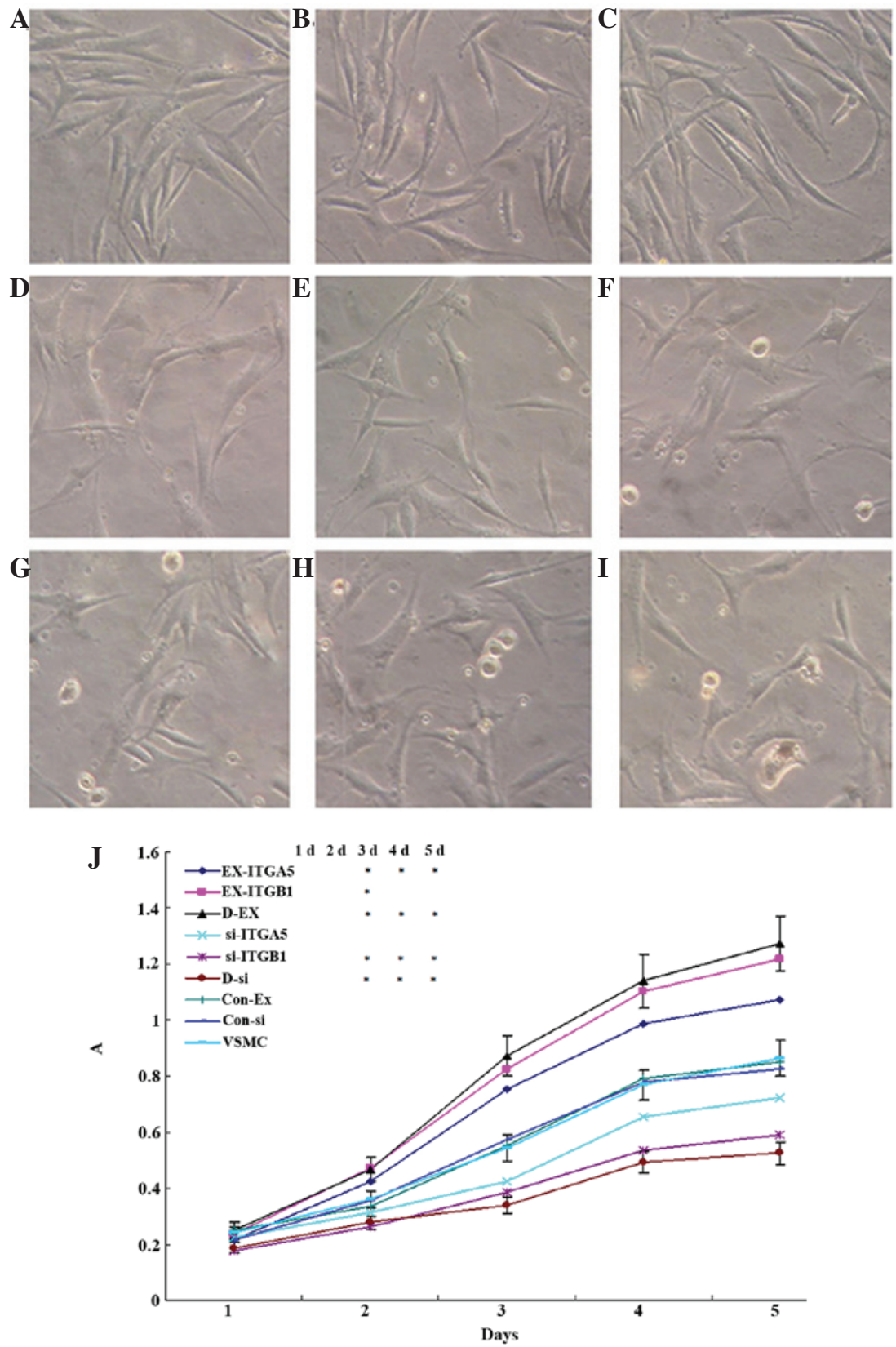

Figure 4. Effects of ITG $\alpha 5$ and ITG $\beta 1$ regulation on the proliferation of VSMCs. (A-I) VSMC morphology visualized under an inverted microscope (magnification, $\mathrm{x} 400$ ). The following cell groups were visualized: (A) EX-ITG $\alpha$ 5, (B) EX-ITG $\beta 1$, (C) D-EX, (D) VSMC, (E) Con-Ex, (F) Con-si, (G) si-ITG $\alpha 5$, (H) si-ITG $\beta 1$, (I) D-si. (J) Effects of ITG $\alpha 5$ and ITG $\beta 1$ regulation on the proliferation of VSMCs, as determined by MTT assay. Values are expressed as the mean \pm standard error of the mean ( $\mathrm{n}=3)$. " $\mathrm{P}<0.05$ vs. VSMC. ITG, integrin; VSMC, vascular smooth muscle cell; si, small interfering; Con, control; EX, overexpressing; A, absorbance.

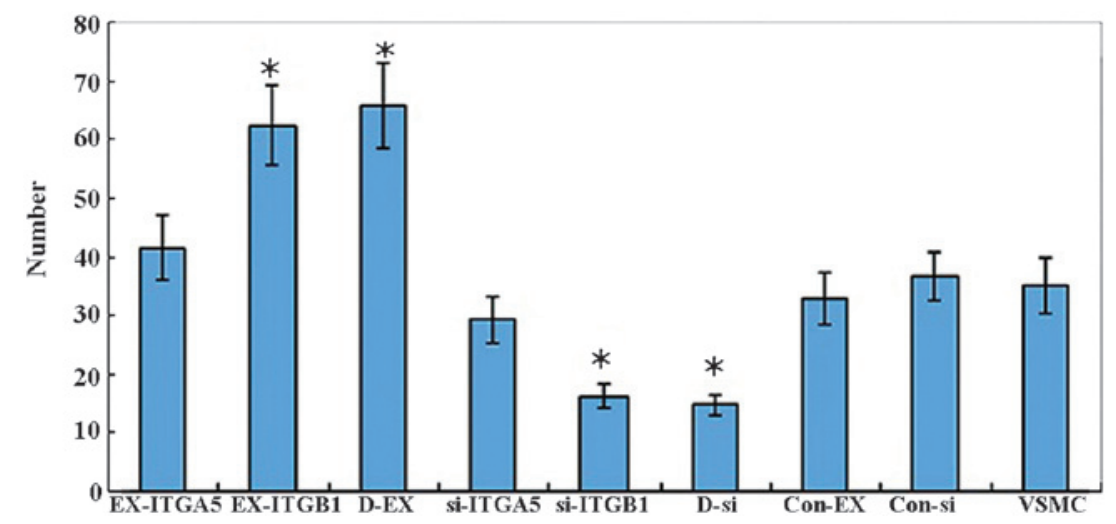

Figure 5. Effects of ITG $\alpha 5$ and ITG $\beta 1$ regulation on the invasion of VSMCs. Values are expressed as the mean \pm standard error of the mean ( $\mathrm{n}=3$ ). ${ }^{*} \mathrm{P}<0.05 \mathrm{vs.}$ VSMC group. ITG, integrin; VSMC, vascular smooth muscle cells; si, small interfering; Con, control; EX, overexpressing. 

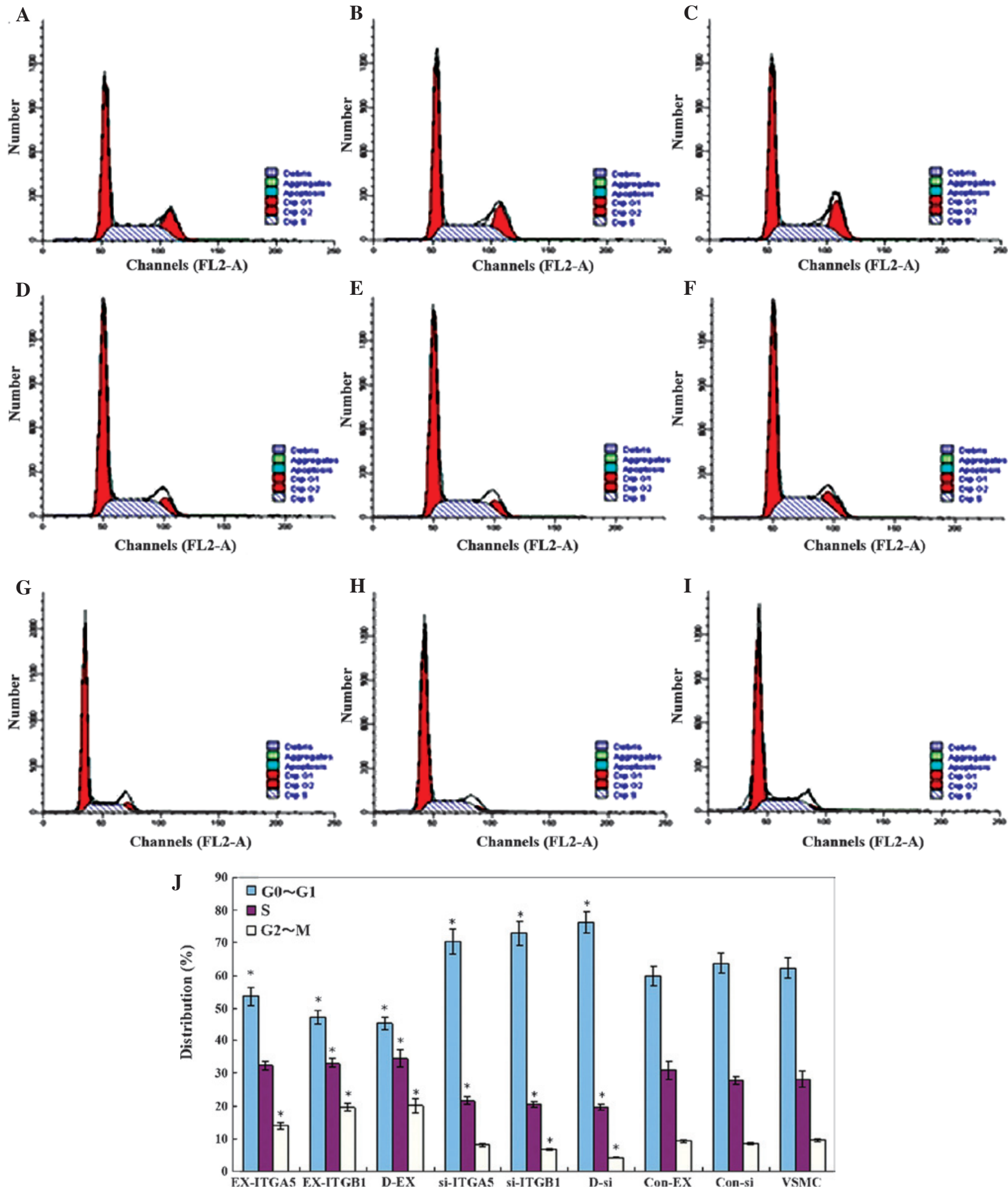

Figure 6. Effects of ITG $\alpha 5$ and ITG $\beta 1$ regulation on the cell cycle of VSMCs. Cell cycle distribution was analyzed in the following groups: (A) EX-ITG $\alpha$, (B) EX-ITG $\beta 1$, (C) D-EX, (D) VSMC, (E) Con-Ex, (F) Con-si, (G) si-ITGa5, (H) si-ITGß1 and (I) D-si. (J) Quantification of cell cycle distribution. Values are expressed as the mean \pm standard error of the mean $(n=3)$. "P<0.05 vs. VSMC group. ITG, integrin; si, small interfering; Con, control; EX, overexpressing; VSMC, vascular smooth muscle cell.

in 96-well reaction plates. The thermocycling conditions were as follows: $95^{\circ} \mathrm{C}$ for $10 \mathrm{~min}$ followed by $95^{\circ} \mathrm{C}$ for $10 \mathrm{sec}$ and $60^{\circ} \mathrm{C} 60 \mathrm{sec}$ for 40 cycles. Gene expression was quantified by $2^{-\Delta \Delta \mathrm{CT}}$ method (15). Relative mRNA levels for each gene were normalized, and values are expressed as the ratio of target gene mRNA to GAPDH expression.
Statistical analysis. Values are expressed as the mean \pm standard deviation. Statistical analyses of differences between the groups were performed using Student's t-test and analysis of variance. SPSS 17.0 (SPSS, Inc., Chicago, IL, USA) was used for all statistical analyses. $\mathrm{P}<0.05$ was considered to indicate a statistically significant difference. 


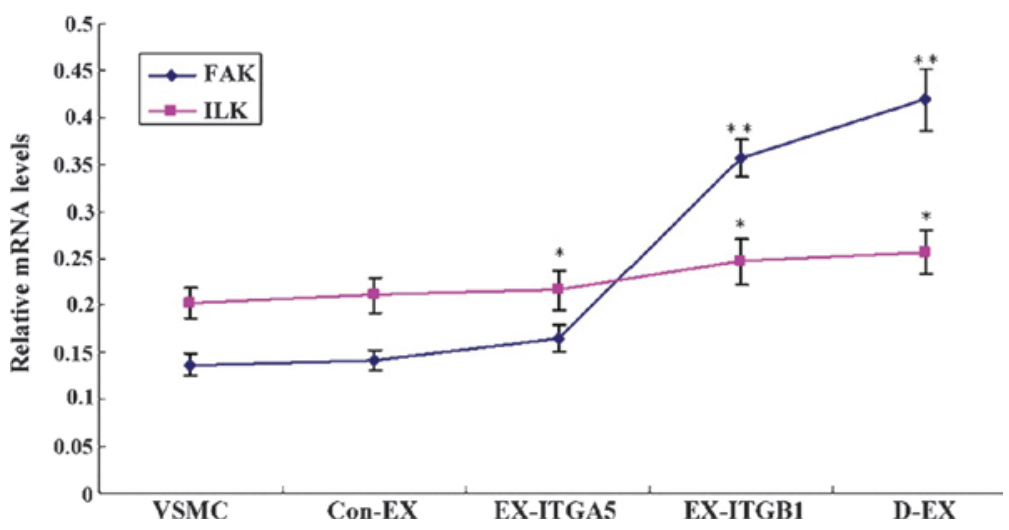

Figure 7. Effects of ITG $\alpha 5$ and ITG $\beta 1$ upregulation on the mRNA expression levels of FAK and ILK in VSMCs. Values are expressed as the mean \pm standard error of the mean ( $\mathrm{n}=3)$. " $\mathrm{P}<0.05$ vs. VSMC group. ${ }^{* *} \mathrm{P}<0.01$ vs. control groups. ITG, integrin; ILK, integrin-linked kinase; FAK, focal adhesion kinase; Con, control; EX, overexpressing; VSMC, vascular smooth muscle cell.

\section{Results}

Construction of overexpression and RNAi vectors. The results of PCR, enzyme analysis and DNA sequencing analysis confirmed that the ITG $\alpha 5$ and ITG $\beta 1$ genes had been successfully cloned (full length, 3,150+176 bp and 2,397+176 bp, respectively), and the pGEM-T-ITG $\alpha 5$ and pGEM-T-ITG $\beta 1$ recombinant clones were confirmed by gene sequencing analysis. Enzyme analysis and gene sequencing indicated that the lentiviral expression vectors Lentivirus-ITG $\alpha 5$ and -ITG $\beta 1$ had been successfully constructed. PCR and DNA sequencing demonstrated that the lentivirus RNAi vectors psiRNA-ITG $\alpha 5$ and -ITG $\beta 1$ were also successfully constructed (Fig. 1). The resulting lentiviral vectors were named pRNAT-U6.2/Lenti-siITG $\alpha 5$ and pRNAT-U6.2/Lenti-siITG $\beta 1$. The viral titers were all $>7.7 \times 10^{5} \mathrm{IU} / \mathrm{ml}$, and were determined by detecting the expression levels of GFP.

Confirmation of transfected cell lines. As shown in Fig. 2A, the mRNA expression levels of ITG $\alpha 5$ and ITG $\beta 1$ were measured by RT-qPCR. The expression levels in the control groups were as follows: Con-si, ITG $\alpha 5$ mRNA $(0.252 \pm 0.026)$ and ITG $\beta 1 \mathrm{mRNA}(0.516 \pm 0.056)$; Con-Ex, ITG $\alpha 5$ mRNA $(0.251 \pm 0.021)$ and ITG $\beta 1$ mRNA $(0.505 \pm 0.062)$; and Con, ITG $\alpha 5(0.238 \pm 0.021)$ and ITG $\beta 1(0.471 \pm 0.051)$. There were no significant differences between the expression levels in the control groups $(\mathrm{P}>0.05)$. The mRNA expression levels of ITG $\alpha 5$ in the EX-ITG $\alpha 5$ and D-EX cells were significantly increased $(\mathrm{P}<0.05)$, and were significantly decreased in the si-ITG $\alpha 5-1$ and $\mathrm{D}$-si cells $(\mathrm{P}<0.05)$, as compared with those in the control groups. In addition, the mRNA expression levels of ITG $\beta 1$ in the EX-ITG $\beta 1$ and D-EX cells were significantly increased $(\mathrm{P}<0.05)$, while being significantly decreased in the si-ITG $\beta 1-1$ and $\mathrm{D}$-si cells $(\mathrm{P}<0.05)$. The same trend was observed with regards to the protein expression levels of ITG $\alpha 5$ and ITG $\beta 1$, as detected by western blot analysis (Fig. 2B). As observed under an inverted microscope, the EX-ITG $\alpha 5$, EX-ITG $\beta 1$ and D-EX cells grew faster, as compared with the control cells, which exhibited better cell morphology and were spindle-shaped. The cells of the si-ITG $\alpha 5$, si-ITG $\beta 1$ and D-si groups had a similar morphology to that of the control cells; however, their growth rate was slightly slower as compared with that of the control cells (Fig. 3).

Effects of ITG $\alpha 5$ and ITG $\beta 1$ on cell growth. Fig. 4 shows the proliferative activity of the cells. There were no significant differences between the control groups, and on the second day there were no significant differences in cell proliferation between any of the groups. From the third day, the absorbance values of MTT were significantly increased in the EX-ITG $\beta 1$ and $\mathrm{D}-\mathrm{EX}$ cells $(\mathrm{P}<0.05)$, and were significantly decreased in the si-ITG $\beta 1$ and $\mathrm{D}-\mathrm{Si}$ cells $(\mathrm{P}<0.05)$, as compared with those in the control group; however, there was no significant difference between the EX-ITG $\alpha 5$ and si-ITG $\alpha 5$ cells $(\mathrm{P}>0.05)$. These results suggested that upregulation of ITG $\beta 1$ expression is able to induce cell proliferation, and downregulation of ITG $\beta 1$ expression may hinder cell proliferation. By contrast, ITG $\alpha 5$ expression had no effect on proliferation of the cells.

Effects of ITG $\alpha 5$ and ITG $\beta 1$ on cell migration. A Transwell chamber migration assay was used to measure the number of cells that passed through an artificial basement membrane (Fig. 5). The numbers of migrated cells in the control

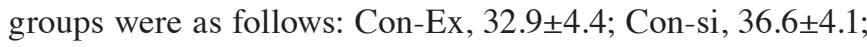
and Con, $35.2 \pm 4.7$, and there was no statistical difference between them. There was no difference between the number of migrated cells in the EX-ITG $\alpha 5(41.5 \pm 5.6)$ and si-ITG $\alpha 5$ $(29.3 \pm 3.9)$ groups, as compared with that in the control groups $(\mathrm{P}>0.05)$; however, the number of migrated cells in the EX-ITG $\beta 1(62.3 \pm 6.8)$ and D-EX (65.7 \pm 7.2$)$ groups was markedly increased $(\mathrm{P}<0.05)$. In addition, the number of migrated cells in the si-ITG $\beta 1(16.2 \pm 2.1)$ and D-si $(14.8 \pm 1.7)$ groups was significantly decreased $(\mathrm{P}<0.05)$. These results indicated that ITG $\beta 1$ may affect the migratory ability of VSMCs. However, ITG $\alpha 5$ had no effect on VSMC cell migration.

Effects of ITG $\alpha 5$ and ITG $\beta 1$ on the cell cycle distribution. The results of the flow cytometric cell cycle analysis showed that, among the three control groups, the proportion of cells in $G_{0} / G_{1}, G_{2} / M$ and $S$ phase were similar ( $P>0.05$; Fig. 6 ). There was a higher proportion of cells in $G_{0} / G_{1}$ phase, as 
compared with that in $S$ phase and $\mathrm{G}_{2} / \mathrm{M}$ phase. In the EX-ITGa5, EX-ITG $\beta 1$ and D-EX groups, the number of cells in $\mathrm{G}_{0} / \mathrm{G}_{1}$ phase was lower, whereas the number of cells in $\mathrm{S}$ phase was increased compared with that in the control groups. In addition, there was a higher proportion of cells in $\mathrm{G}_{0} / \mathrm{G}_{1}$ phase in the si-ITG $\alpha 5$, si-ITG $\beta 1$ and D-si groups, and a lower proportion of cells in $\mathrm{S}$ and $\mathrm{G}_{2} / \mathrm{M}$ phase as compared with that in the control groups. These results suggested that upregulation of ITG $\beta 1$ expression may be able to induce cell division.

Effects of ITG $\alpha 5$ and ITG $\beta 1$ on the mRNA expression levels of ILK and FAK. The expression levels of ILK in the control groups were as follows: Con-Ex $(0.142 \pm 0.011)$, Con-si $(0.129 \pm 0.012)$ and Con $(0.137 \pm 0.012)$, and there were no differences detected between them $(\mathrm{P}>0.05)$. The mRNA expression levels of FAK in the EX-ITG $\beta 1(0.357 \pm 0.0194)$ and D-EX cells $(0.419 \pm 0.033)$ were significantly increased as compared with those in the control groups $(\mathrm{P}<0.01$; Fig. 7). The mRNA expression levels of FAK in the si-ITG $\beta 1$ $(0.054 \pm 0.008)$ and $\mathrm{D}$-si $(0.034 \pm 0.004)$ cells were decreased compared with those in the control $(\mathrm{P}<0.01)$. There were no marked changes in ILK mRNA expression levels between the groups. These results indicated that ITG $\beta-1$ may be a major regulator of FAK mRNA expression.

\section{Discussion}

In the present study, six types of transfected VSMCs were successfully constructed: and ITG $\alpha 5$-overexpressing cell line (EX-ITG $\alpha 5)$, an ITG $\beta 1$-overexpressing cell line (EX-ITG $\beta 1$ ), an ITG $\alpha 5$ - and ITG $\beta 1$-overexpressing cell line (D-EX), an ITG $\alpha 5$ knockdown cell line (si-ITG $\alpha 5$ ), an ITG $\beta 1$ knockdown cell line (si-ITG $\beta 1$ ) and an ITG $\alpha 5$ - and ITG $\beta 1$-knockdown cell line (D-si). A pLentiGFP empty vector cell line (Con-Ex), pRNAT-U6.2/Lenti empty vector cell line (Con-si) and normal VSMC cell line were also used, alongside the six experimental cell lines, to analyze cell cycle distribution, proliferation and migration.

The results of the present study demonstrated that ITG $\beta 1$ upregulation was able to induce VSMCs to re-enter the cell division cycle, and increase proliferation and migration. Conversely, downregulation of ITG $\beta 1$ resulted in the opposite effect in VSMCs. Furthermore, ITG $\alpha 5$ regulation had no effect on the proliferation and migration of VSMCs. Abnormal proliferation and migration of VSMCs is the main cause of early vascular restenosis (16). Integrins are a family of glycoprotein cell surface receptors that participate in cell adhesion with the ECM, and have an important role in regulating cell growth, differentiation and proliferation. ITG $\alpha 5 \beta 1$ is associated with the migration, proliferation and vascular injury repair process of VSMCs (17). The present study compared three cell groups: Overexpressing cells, including EX-ITG $\alpha 5$, EX-ITG $\beta 1$ and D-EX; knockdown groups, including si-ITG $\alpha 5$, si-ITG $\beta 1$ and D-si; and the control cells, including Con-Ex, Con-si and normal VSMCs. ITG $\beta 1$ was shown to be able to positively regulate the proliferation and migration of VSMCs and induce cell division. By contrast, ITG $\alpha 5$ did not have any effect on the functions of VSMCs. These results indicated that ITG $\beta 1$ may be involved in the proliferation and migration of VSMCs.
During ITG-mediated proliferation and migration of VSMCs, FAK and ILK were shown to be involved in the signaling pathway. Biochemical evidence has previously suggested that ILK may interact with ITG $\beta 1$ as well as ITG $\beta 3$ (18). ITG $\beta 1$ and the associated ILK have a crucial role in cell survival, tissue homeostasis and carcinogenesis. ILK associates with ITG tails and thereby links ITGs with the actin cytoskeleton and various signaling pathways $(19,20)$. FAK may be the main structural basis for the signal conduction of VSMC proliferation and migration regulation (2). The results of the present study showed that the mRNA expression levels of FAK in the EX-ITG $\beta 1$ and D-EX cells were increased, whereas they were decreased in the si-ITG $\beta 1$ and D-si cells. However, there were no marked changes in ILK mRNA expression levels. These results suggested that ITG $\beta 1$ may be a major regulator of FAK mRNA expression.

In conclusion, the present study investigated the role of ITG $\alpha 5 \beta 1$ in the proliferation and migration of VSMCs. ITG $\beta 1$ was shown to be involved in the proliferation and migration of VSMCs, and FAK was involved in the signaling pathways of ITG $\beta 1$. These results may provide a possible therapeutic target for the prevention and treatment of VSMC-associated early vascular disease.

\section{Acknowledgements}

The present study was supported by a grant from the Basic and Advanced Technology Research Projects of Henan Province, China (no. 1223004110184).

\section{References}

1. Han M, Wen JK, Zheng B, Liu Z and Chen Y: Blockade of integrin beta3-FAK signaling pathway activated by osteopontin inhibits neointimal formation after balloon injury. Cardiovasc Pathol 16: 283-290, 2007.

2. Huang S, Sun Z, Li Z, Martinez-Lemus LA and Meininger GA: Modulation of microvascular smooth muscle adhesion and mechanotransduction by integrin-linked kinase. Microcirculation 17: 113-127, 2010.

3. Ruoslahti E: Fibronectin and its receptors. Annu Rev Biochem 57: 375-413, 1988.

4. Pickering JG, Chow LH, Li S, Rogers KA, Rocnik EF, Zhong R and Chan BM: alpha5beta1 integrin expression and luminal edge fibronectin matrix assembly by smooth muscle cells after arterial injury. Am J Pathol 156: 453-465, 2000.

5. Li JJ, Han M, Wen JK and Li AY: Osteopontin stimulates vascular smooth muscle cell migration by inducing FAK phosphorylation and ILK dephosphorylation. Biochem Biophys Res Commun 356: 13-19, 2007.

6. Koshman YE, Engman SJ, Kim T, Iyengar R, Henderson KK and Samarel AM: Role of FRNK tyrosine phosphorylation in vascular smooth muscle spreading and migration. Cardiovasc Res 85: 571-581, 2010.

7. Brakebusch $C$ and Fässler R: The integrin-actin connection, an eternal love affair. EMBO J 22: 2324-2333, 2003.

8. Zhang YG, Guo X, Zhou JJ, Yu B and Liu B: [Sorting of packaging cells for retroviral vector carrying green fluorescent gene and viral titer determination]. Di Yi Jun Yi Da Xue Xue Bao 25: 30-32, 36, 2005 (In Chinese).

9. Zhou Y, Luo W, Zheng L, Li M and Zhang Y: Construction of recombinant FGFR1 containing full-length gene and its potential application. Plasmid 64: 60-67, 2010.

10. Adams LS, Phung S, Yee N, Seeram NP, Li L and Chen S: Blueberry phytochemicals inhibit growth and metastatic potential of MDA-MB-231 breast cancer cells through modulation of the phosphatidylinositol 3-kinase pathway. Cancer Res 70: 3594-3605, 2010. 
11. Wang Z, Banerjee S, Li Y, Rahman KM, Zhang Y and Sarkar FH: Down-regulation of notch-1 inhibits invasion by inactivation of nuclear factor-kappaB, vascular endothelial growth factor, and matrix metalloproteinase-9 in pancreatic cancer cells. Cancer Res 66: 2778-2784, 2006.

12. Zhang Y, Zhang J, Dai B, Wang N and He L: Anti-proliferative and apoptotic effects of the novel taspine derivative tas 41 in the Caco-2 cell line. Environ Toxicol Pharmacol 31: 406-415, 2011.

13. Zhang YM, Dai BL, Zheng L, et al: A novel angiogenesis inhibitor impairs lovo cell survival via targeting against human VEGFR and its signaling pathway of phosphorylation. Cell Death Dis 3: e406, 2012.

14. Zhang Y, Zheng L, Zhang J, Dai B, Wang N, Chen Y and He L: Antitumor activity of taspine by modulating the EGFR signaling pathway of Erk1/2 and Akt in vitro and in vivo. Planta Med 77: 1774-1781, 2011.

15. Livak KJ and Schmittgen TD: Analysis of relative gene expression data using real-time quantitative PCR and the 2(-Delta Delta C(T)) Method. Methods 25: 402-408, 2001.
16. Shi L, Ji Y, Jiang X, et al: Liraglutide attenuates high glucose-induced abnormal cell migration, proliferation, and apoptosis of vascular smooth muscle cells by activating the GLP-1 receptor, and inhibiting ERK1/2 and PI3K/Akt signaling pathways. Cardiovasc Diabetol 14: 18, 2015.

17. Friedrich EB, Clever YP, Wassmann S, Werner N, Böhm M and Nickenig G: Role of integrin-linked kinase in vascular smooth muscle cells: Regulation by statins and angiotensin II. Biochem Biophys Res Commun 349: 883-889, 2006.

18. Hannigan GE, Leung-Hagesteijn C, Fitz-Gibbon L, et al: Regulation of cell adhesion and anchorage-dependent growth by a new beta 1-integrin-linked protein kinase. Nature 379: 91-96, 1996.

19. Böttcher RT, Lange A and Fässler R: How ILK and kindlins cooperate to orchestrate integrin signaling. Curr Opin Cell Biol 21: 670-675, 2009.

20. Kanasaki K, Kanda Y, Palmsten K, et al: Integrin beta1-mediated matrix assembly and signaling are critical for the normal development and function of the kidney glomerulus. Dev Biol 313: 584-593, 2008 